\title{
Resuscitation refresher training in the wake of the COVID-19 pandemic - instructor-led training outperforms self-directed learning.
}

\author{
Alice O'Donnell \\ University of Birmingham College of Medical and Dental Sciences \\ Aniela Pawar \\ University of Birmingham College of Medical and Dental Sciences \\ Emma Hardy \\ University of Birmingham College of Medical and Dental Sciences \\ Harriet Percival \\ University of Birmingham College of Medical and Dental Sciences \\ Nicholas Coffin \\ Department of Anaesthesia and Intensive Care Medicine, Salford Royal Hospital, UK \\ Jonathan Hulme
}

Department of Anaesthesia and Intensive Care Medicine, Sandwell and West Birmingham NHS Trust, UK

\section{Andrew Owen}

Department of Anaesthesia and Critical Care Medicine, Queen Elizabeth Hospital, Birmingham Joseph Alderman ( $\square$ joseph.alderman@nhs.net)

https://orcid.org/0000-0001-8273-9009

\section{Original research}

Keywords: Basic life support, BLS, Cardiopulmonary resuscitation, CPR, refresher training, video assisted learning, self directed learning, Covid-19

Posted Date: October 15th, 2020

DOI: https://doi.org/10.21203/rs.3.rs-61530/v2

License: @ (i) This work is licensed under a Creative Commons Attribution 4.0 International License. Read Full License 


\section{Abstract}

Background: survival following out-of-hospital cardiac arrest is worse in the United Kingdom than in many other developed countries, possibly due to below average delivery of early bystander cardiopulmonary resuscitation (CPR). Training laypeople how to perform CPR is vital, but skill decays quickly. Most methods of training require face-to-face contact which is challenging during the Covid-19 pandemic. The optimal method of delivering CPR refresher training is not yet known. Our aim was to understand how basic life support (BLS) performance decays over time, and how best to deliver refresher training in terms of timeframe and training method - are methods with limited instructor / student contact effective?

Methods: this quasi-randomised trial allocated participants to receive refresher training one, two, or three years after their initial BLS training. Participants' baseline BLS performance was assessed by a trained instructor, and then they were randomly allocated to receive refresher training via one of three methods: oneto-one instructor led training, video assisted learning with self-directed practice, or a written information guide alone. BLS performance was assessed again following refresher training.

Results: 42 participants were recruited - all completed all study stages. Baseline BLS performance was poor only $21 \%$ of participants passed a formative assessment. Students who had received BLS training most recently showed better BLS performance than those where greater time had elapsed. one-to-one training was the most effective refresher training modality, and video assisted training did not lead to significant improvement in BLS performance.

Conclusion: one-to-one BLS refresher training outperforms self-directed methods with more limited instructor contact, though it requires greater resource and planning. It may be impractical to deliver instructor-led BLS refresher training during the Covid-19 pandemic. The video we chose to use for our training was not interactive, though such tools exist. Alternative video-led approaches (with or without instructor guidance) should be explored to investigate if they are more effective than the tool we used in this study.

\section{Background}

The United Kingdom lags behind other similarly developed countries in terms of outcomes for patients following Out of Hospital Cardiac Arrest (OHCA). In England, only 7-8\% of patients survive OHCA (1), yet bystander cardiopulmonary resuscitation (CPR) is commenced in just $40-50 \%$ of cases (2). Bystander CPR rates, and subsequent survival from OHCA is higher in many other developed countries than the UK, resulting in leading health organisations calling for an increase in awareness and knowledge of CPR and how it should be performed. $(4,5)$

CPR is a crucial element in the chain of survival following OHCA and thorough teaching of correct techniques to both the general public and healthcare professionals can save lives. (5) Given their future clinical roles, it is 
crucial that healthcare students are competent at delivering CPR both during their time in training, and throughout their professional lives. Optimal models for CPR training are still debated, with divergent curricula and training manikins used internationally. Whilst international guidelines exist recommending rates and depth of chest compression, ventilation ratios and times, and duty cycle $(6,7)$, debate exists about how to best teach these guidelines.

A growing literature demonstrates that CPR skills decay over time, with skill retention in this field being described as 'very poor' and 'not meeting the required standard' 12 months post training. (8-12) A 2014 study looking at retention of first aid and Basic Life Support (BLS) skills in undergraduate medical students found that $82 \%$ of students failed a BLS assessment 1-year post training. (9) Moreover, even amongst trained and experienced rescuers, the quality of CPR delivered in real-world scenarios is variable, and often does not meet international guidelines. (10)

At the end of 2019 a novel coronavirus - Severe Acute Respiratory Syndrome Coronavirus 2 (SARS-CoV-2) emerged in Hubei province, China, and has subsequently spread as a pandemic. $(13,14)$ Most infections are asymptomatic or mild, however a significant proportion develop severe respiratory disease - Covid-19, requiring hospitalisation for respiratory support. Current best evidence suggests that both airborne and fomite transmission are possible means of viral spread, though recent evidence suggests the former predominates. (15) Infection prevention and control measures have been variably adopted by Countries worldwide; current World Health Organisation guidelines recommend 1 metre social distancing, meticulous hand hygiene, avoiding large gatherings, and self-isolation if symptomatic. (16) The utility of face coverings is still debated, though there is no evidence they cause harm and may have limited benefit in preventing transmission from asymptomatic carriers. (17) This new and highly infectious illness has serious implications for the normal functioning of human society.

The impact of Covid-19 on healthcare has been profound, impairing previously well-functioning health systems' delivery of both routine and emergency care. Adding to the mortality directly attributable to Covid-19, treatment and outcomes for other conditions worsened during the peak of the pandemic. Survival from OHCA deteriorated in one report from Paris, France (18) - bystander CPR rates were lower - suggesting that members of the public were understandably reticent to deliver BLS to strangers. Life support training will need to adapt to give would-be rescuers the confidence to safely administer effective CPR in the context of a respiratory viral pandemic.

Life support education at the University of Birmingham is delivered in a peer-peer model, with peer-led education supervised by a faculty of medical practitioners. (19) At present, Basic Life Support (BLS) training is delivered in the first year of the undergraduate medical curriculum, and not routinely refreshed until employment post-graduation. Prior to the emergence of Covid-19, training was delivered in face-to-face tutorials, with groups of 6-12 students trained by three instructors. Equipment was shared, but surfaces decontaminated between uses. Methods being planned to reduce Covid-19 transmission risk include reduced group sizes, hand hygiene, and greater reliance on remote or participant-led training in lieu of face-to-face training. 
This study aimed to address when and how to deliver BLS refresher training for undergraduate healthcare students, and to assess the use of participant-led refresher training methods as opposed to one-to-one instructor led training.

\section{Methods}

This quasi-randomised trial was designed in three phases: 1 - assessing CPR skills of medical students at least one year after their BLS training; 2 - refreshing their skills using variable methods; 3 - re-assessment of their CPR skills.

Potential participants were identified from the undergraduate medical student cohort at the University of Birmingham, and were screened against inclusion and exclusion criteria. Eligible participants were given written information about the study prior to recruitment and asked to provide written informed consent for participation in the study. Inclusion and exclusion criteria for this study are as follows:

\section{Inclusion criteria:}

- Current medical student at the University of Birmingham in $2^{\text {nd }}, 3^{\text {rd }}$ or $4^{\text {th }}$ year of study.

- Successfully passed a BLS course delivered by the University of Birmingham during semester 1 of their first year of medical training.

- Written informed consent to participate after receiving a participant information sheet.

\section{Exclusion criteria:}

- Students involved in course administration, teaching or examining for our training network, or linked groups.

- Instructor trainer qualification for any life support, first aid or lifeguarding support.

- New or chronic injury which may adversely affect CPR performance.

Phase 1 involved assessing the CPR performance of medical students at one, two, or three years following their BLS training (dependent on when they had been trained, and their current year of study). Participants were asked to perform BLS on a Laerdal Little Anne® manikin and were assessed by one of two trained assessors using the BLS assessment tool used at the University of Birmingham. This tool uses a simple tickbox form based on European Resuscitation Council (ERC) guidelines, designed by our team to achieve repeatable results, and in wide use in our organisation. (20) The facilitators then recorded the results of their initial assessment.

During Phase 2, participants were assigned randomly into groups and received refresher BLS training in a 30minute tutorial using one of three methods:

a. One-to-one training from an ERC accredited BLS instructor. The instructor was the same for all participants in this group, and was blinded to the results of the candidates' initial assessments. The participants had full access to Laerdal Little Anne manikin for hands-on practice throughout their tutorial. 
b. Video assisted training with the opportunity for self-guided practice. Participants were placed alone in a room and then shown a short video detailing the BLS algorithm, created by the British Heart Foundation for their HeartStart training. (21) They could use the remaining time to practice with a Laerdal Little Anne manikin as much or as little as they liked.

c. Written BLS information only. Participants sat alone in a room and were provided with a written information sheet with details of the ERC BLS algorithm, to read for as much or as little as they liked.

Phase 3 involved reassessment of BLS performance following refresher training. Participants were reassessed by the same facilitator, using the same assessment tool as in Phase 1. The facilitators were blinded to the participants' refresher training method assignment. The facilitators recorded the results of each participant's BLS assessment. Groups of participants were prevented from mixing until after the study had concluded.

Sample size was calculated as 8 participants per refresher training modality subgroup based on prior internal pilot data (not shown). Pass rates are expressed as number (percentage), and were compared between groups using 2-tailed Fisher's exact test on the original categorical data. A p value of 0.05 was used as the limit of statistical significance. All data were analysed using $R$ version 4.0.2. (22)

\section{Results}

42 students were recruited for this study (see Fig. 1).

All students were assessed before and after refresher training. Baseline characteristics for all participants are shown in Table 1.

Table 1: Baseline characteristics of participants

\begin{tabular}{|lll|}
\hline & Count & $\%$ \\
\hline Gender: & 11 & $26.2 \%$ \\
Male & 31 & $73.8 \%$ \\
\hline Yemale & & \\
1 & 13 & $31.0 \%$ \\
2 & 11 & $26.2 \%$ \\
3 & 18 & $42.8 \%$ \\
\hline
\end{tabular}

Prior to refresher training, compliance with ERC BLS guidelines was initially $21 \%$. The students at one year post their initial training had the highest pre-refresher pass rate of $38.5 \%$. Students who were assessed at two and three years post training had lower pre refresher pass rates, at $9.1 \%$ and $16.7 \%$ respectively. 
Following refresher training BLS quality improved - compliance with ERC BLS guidelines rose from $23.8 \%$ to $61.9 \%$ ( $p=0.0008$, Fisher's exact test). Overall, the pass rate in each group increased after the refresher training (Fig. 2).

The

Table 2: Breakdown of BLS performance before and after refresher training (measured as compliance with ERC BLS guidelines). Broken down by time elapsed since initial BLS training. Comparisons are presented between groups of participants who were 1,2 and 3 years after their initial BLS training; and for each group individually in terms of pre-refresher and post-refresher performance. $P$ values were calculated using Fisher's Exact Test.

\begin{tabular}{|c|c|c|c|c|}
\hline & $\begin{array}{l}1 \text { year after } \\
\text { training } \\
n=13\end{array}$ & $\begin{array}{l}2 \text { years after } \\
\text { training } \\
n=11\end{array}$ & $\begin{array}{l}3 \text { years after } \\
\text { training } \\
n=18\end{array}$ & $\begin{array}{l}1 \text { vs } 2 \text { vs } 3 \text { years after } \\
\text { initial training }\end{array}$ \\
\hline Pre-refresher pass rate & $5(34.5 \%)$ & $2(9.1 \%)$ & $3(16.7 \%)$ & $p=0.34$ \\
\hline Post-refresher pass rate & $7(53.9 \%)$ & $7(63.6 \%)$ & $12(66.7 \%)$ & $p=0.85$ \\
\hline $\begin{array}{l}\% \text { change in pass rate } \\
\text { following refresher training }\end{array}$ & $\begin{array}{l}18.6 \% \\
p=0.70\end{array}$ & $\begin{array}{l}54.4 \% \\
p=0.08\end{array}$ & $\begin{array}{l}50 \% \\
p=0.006\end{array}$ & \\
\hline
\end{tabular}

Comparison between different refresher training modalities is displayed in Table 3. One-to-one training outperformed the other two modalities, with candidates refreshed in this way displaying an increase in pass rate from $33.3 \%$ to $100 \%$ ( $p=0.0090$, Fisher's exact test). Refresher training using an information sheet also led to a significant increase in pass rate from $18.8 \%$ to $56.3 \%$ ( $p=0.0290$, Fisher's exact test).

Table 3: Breakdown of compliance with ERC BLS guidelines after refresher training by modality of training. Comparisons are presented between groups based on the method of refresher training they received; and for each group individually in terms of pre-refresher and post-refresher performance. $P$ values were calculated using Fisher's Exact Test.

\begin{tabular}{|lllll|}
\hline & $\begin{array}{l}\text { One-to-one } \\
\text { instructor }\end{array}$ & $\begin{array}{l}\text { Video } \\
\text { assisted }\end{array}$ & $\begin{array}{l}\text { Information } \\
\text { sheet }\end{array}$ & $\begin{array}{l}\text { One-to-one vs video assisted } \\
\text { vs information sheet }\end{array}$ \\
\hline $\mathbf{n = 9}$ & $3(33.3 \%)$ & $\begin{array}{l}3 \\
(17.7 \%)\end{array}$ & $3(18.8 \%)$ & $\mathrm{n}=16$ \\
\hline Pre-refresher pass rate & $9(100.0 \%)$ & $\begin{array}{l}7 \\
(41.2 \%)\end{array}$ & $10(56.3 \%)$ & $\mathrm{p}=0.009$ \\
\hline Post-refresher pass rate & $66.7 \%$ & $23.5 \%$ & $37.5 \%$ & \\
$\begin{array}{l}\text { \% change in pass rate } \\
\text { following refresher training }\end{array}$ & $\mathrm{p}=0.009$ & $\mathrm{p}=0.26$ & $\mathrm{p}=0.03$ & \\
\hline
\end{tabular}




\section{Discussion}

This study highlights two important points: BLS skill decays rapidly in previously competent medical students, and one-to-one instruction is the optimal way to enhance refresher learning.

The decay in BLS performance worsens as time elapsed since the original BLS training increases, with the pass rate (compliance with ERC BLS guidelines during formal assessment by a trained examiner) decreasing to $34.5 \%$ even 1 year following the primary course and declining further thereafter. Such findings highlight that in order to prevent significant skill decay, refresher training should commence at some stage within the first 12 months of initial BLS teaching. Of note, as students in our institution become more senior they move from lecture theatres to clinical placements, and are more likely to be exposed to cardiac arrests in hospital. Many students in their fourth year of study (three years following initial BLS training) receive CPR training in their hospital. This may account for their small improvement in pre-refresher training BLS performance when compared to students 2 years after their initial training.

Our study also investigated the most effective method of refresher teaching. We found that one-to-one training between student and instructor served as the most effective means, with post-refresher training compliance during skill assessment meeting ERC guidelines in $100 \%$ of individuals re-assessed. The use of an information sheet alone improved BLS performance, albeit to a lesser-degree than one-to-one training, and sole use of video-assisted training showed an even smaller improvement in BLS performance (nonsignificant).

Each method of refresher training carries advantages and disadvantages. Although one-to-one teaching incurs the best improvement in post-refresher competency, it is the most resource-heavy option, requiring the presence of suitably qualified instructors. In our study, we trained students in a 1:1 ratio with instructors larger instructor to student ratios may allow greater efficiency, and reduced resource use. That said, larger group sizes increase risk of Covid-19 exposure, and safely moving large groups into and out of training venues whilst practicing social distancing will be challenging.

In contrast, video-assisted training with the opportunity for self-practice requires less resource, and is much less likely to encourage Covid-19 transmission. The use of a pre-recorded video offers a reproducible alternative to one-to-one instruction. Large numbers of students are able to watch the video at once and resources could easily be disseminated via an online platform for quick and easy access. Use of technology in this way may be a scalable refresher training option for centres with limited resources or larger cohorts. Disadvantages of video-assisted training include the lack of instructor interaction with students and the subsequent inability to ask questions in order to further understanding and consolidate knowledge. Similarly, without having a qualified instructor present to observe practice, students are unable to receive feedback on their performance and they are left to self-determine their competence. In our study, video-assisted refresher training as a sole modality failed to meaningfully improve candidates' performance. Though high quality, the British Heart Foundation's Heartstart video we used during training only runs for 6 minutes and is not interactive - it is intended to be followed by group or individual hands-on practice. An alternative interactive option is the Lifesaver video produced by the Resuscitation Council (UK). (23) A randomised controlled trial comparing Lifesaver to face-to-face training in school children demonstrated broadly similar performance 
following initial training, though combination of face-to-face training and Lifesaver outperformed both modalities in isolation. (24) The British Heart Foundation has developed an alternative video curriculum titled "Call Push Rescue" which is designed to be displayed in a classroom with candidates performing CPR together, using their own individual manikins. (25) It was beyond the scope of this study to compare different video modalities, though it is possible candidates' performance would have been different had we chosen to use either Lifesaver or Call Push Rescue.

Participants who received refresher training via a written information sheet to read showed significant improvement in their post-refresher BLS performance, albeit to a lesser extent than seen with one-to-one training. One implication of this method of refresher training is that no Laerdal Little Anne ${ }^{\circledR}$ manikins are required. On one hand, this potentially puts candidates at a disadvantage as they have no opportunity to practice hands-on CPR. On the other hand, it may be a suitable option for those centres who have very limited resources and funds, where manikins are reserved for de novo BLS training only.

Overall, BLS refresher training involving one-to-one teaching with a qualified instructor is the optimal way of refreshing BLS skill in terms of pass rates, yet uses the most resources and therefore may not be suitable for all centres. Given the current Covid-19 pandemic, it may be undeliverable in many contexts. Using videorecordings and written information sheets in combination with - or in lieu of - one-to-one refresher training may allow rapid, effective skill refreshment whilst minimising risk of Covid-19 transmission. It is feasible that skill retention following different refresher training modalities may differ - for instance, participants re-trained using video assisted self-learning may require more frequent refresher sessions than those re-trained using instructor led face-to-face methods. The optimum window in which to schedule further refresher training following each modality of refresher training is a topic for future study.

Our study has several important limitations. Firstly, despite achieving our recruitment target from a power calculation based on pilot data, our relatively small sample size (42) limits the study's generalisability. There are unequal numbers of participants in each refresher group given the increased instructor resource required to run one-to-one training. The use of the same examiner to assess participants both pre- and post-refresher training introduces the risk of observer bias, limiting the value of comparisons drawn from both pre- and postrefresher training. However, blinding with regard to group allocation means that methods of refresher training can be safely compared. Some of the improvement in scores between the initial and post-refresher assessment may be attributable to practice participants obtained in sitting the initial assessment itself, though this would not explain differences observed between groups. Finally, the study is temporally limited with regards the amount of time elapsed since participants' original BLS training. As such, our results may not be generalisable to the wider population outside of the medical student cohort, or to those for whom a greater amount of time has elapsed since their initial BLS training.

Future research should aim to recruit a more representative cross-sectional sample of the overall lay population, specifically including non-healthcare students, members of the public and/or school children. Although our study only investigated three methods of refresher training, it still provides a valuable comparison that highlights clear differences in effectiveness of these methods. This study is the first of its kind on this subject, and clearly demonstrates that BLS skill decays in medical students after as little as one 
year. Additionally, it has shown the variation in efficacy of three different refresher teaching methods that are simple, adaptable and inclusive.

\section{Conclusion}

This study is the first of its kind to investigate how BLS performance decay rapidly over time amongst medical students, and also the optimal way of refreshing BLS skill. When methods of refresher training were compared, one to one teaching was found to be the most effective reteaching method. Although use of written information sheets and video resources are attractive given their scalability, reduced resource requirement and reduced risk of Covid-19 transmission, they are unlikely to be sufficient in isolation.

\section{Declarations}

\section{Ethics approval and consent to participate}

Ethical self-assessment was conducted. This project was delivered as part of an existing locally ethically approved programme of work (University of Birmingham Ethics Committee). Specific project ethical approval was not needed.

$\underline{\text { Consent for publication }}$

Not applicable

Availability of data and materials

The datasets generated and analysed during the current study are not publicly available (because the small number of participants means that participants could be identifiable despite anonymisation). The data are available from the corresponding author on reasonable request.

\section{Competing interests}

The authors declare that they have no competing interests

\section{Funding}

No funding was used or required in the delivery of this project.

\section{Authors' contributions}

- $A O^{\prime} D$ was responsible for: study design, recruitment, consenting participants, recording data, and authoring manuscript.

- AP was responsible for: recruitment, consenting participants, recording data, and reviewing manuscript.

- EH was responsible for: recruitment, consenting participants, recording data, and reviewing manuscript.

- NC was responsible for: reviewing manuscript, and senior supervision of project.

- JH was responsible for: reviewing manuscript, and senior supervision of project. 
- AO was responsible for: authoring manuscript, data analysis, and senior supervision of project.

- JEA was responsible for: study design, authoring manuscript, data analysis, figure preparation, and overall supervision of project.

\section{Acknowledgements}

Not applicable

Authors' information

Not applicable

\section{Abbreviations}

BLS - Basic Life Support

CPR - Cardiopulmonary Resuscitation

ERC - European Resuscitation Council

OHCA - Out of Hospital Cardiac Arrest

SARS-CoV-2 - Severe Acute Respiratory Syndrome Coronavirus 2

\section{References}

1. Perkins GD, Lockey A, de Belder MA et al. National initiatives to improve outcomes from out-of-hospital cardiac arrests in England. Emerg Med J. 2015; 33(7): 448-451

2. Out of Hospital Cardiac Arrest Outcomes (OHCAO) registry. Review of the first 5 years and forward strategy. University of Warwick. [cited 6 Aug 2020]. [Internet]. Available from URL:

https://warwick.ac.uk/fac/sci/med/research/ctu/trials/ohcao/publications/showcase/57904_ctu_reportfinal.pdf

3. British Heart Foundation. Leading organisations call for overhaul of cardiac arrest treatments. 2017 [cited 8 May 2020]. [internet]. Available from: https://www.bhf.org.uk/news-from-the-bhf/newsarchive/2017/march/cardiac-arrest-report

4. Perkins GD, Lockey AS, de Belder MA on behalf of the Community Resuscitation Group, et al. National initiatives to improve outcomes from out-of-hospital cardiac arrest in England. Emerg Med J 2016;33:448-451.

5. Resuscitation council UK. CPR. 2014 [cited 8 May 2020]. [internet]. Available from: https://www.resus.org.uk/faqs/faqs-cpr/

6. Perkins GD, Handley AJ, Koster RW et al. European Resuscitation Council Guidelines for Resuscitation 2015. Section 2. Adult basic life support and automated external defibrillation. Resuscitation. 2015; 95 : 81-99 
7. Kleinman ME, Brennan EE, Goldberger ZD et al. Part 5: Adult Basic Life Support and Cardiopulmonary Resuscitation Quality. 2015 American Heart Association Guidelines for Cardiopulmonary Resuscitation and Emergency Cardiovascular Care. Circulation. 2015; 132:s414-435

8. Sternbach, George L. et al. The retention of cardiopulmonary resuscitation skills. J Emerg Med 1984; 2(1):33-6

9. De Ruijter PA, Biersteker HA, Biert J, van Goor H, Tan EC. Retention of first aid and basic life support skills in undergraduate medical students. Med Educ Online. 2014;19:10.3402/meo.v19.24841. doi:10.3402/meo.v19.24841.

10. Woollard M, Whitfeild R, Smith A, Colquhoun M, Newcombe RG, Vetteer N, et al. Skill acquisition and retention in automated external defibrillator (AED) use and CPR by lay responders: a prospective study. Resuscitation. 2004;60:17-28.

11. Das M, Elzubeir M. First aid and basic life support skills training early in the medical curriculum: curriculum issues, outcomes, and confidence of students. Teach Learn Med. 2001;13:240-6.

12. Edward C. T. H. Tan, Irma Severien, Jaap C. M. Metz, Hubert J. J. M. Berden, Jan Biert. First aid and basic life support of junior doctors: A prospective study in Nijmegen, the Netherlands. Med Teach. 2006;28(2):189-192.

13. Ghinai I, McPherson TD, Hunter JC, Kirking HL, Christiansen D, Joshi K, Rubin R, Morales-Estrada S, Black SR, Pacilli M, Fricchione MJ. First known person-to-person transmission of severe acute respiratory syndrome coronavirus 2 (SARS-CoV-2) in the USA. Lancet. 2020;395:1137-44

14. Guan WJ, Ni ZY, Hu Y, Liang WH, Ou CQ, He JX, Liu L, Shan H, Lei CL, Hui DS, Du B. Clinical characteristics of coronavirus disease 2019 in China. New Eng J Med. 2020;382(18):1708-20.

15. Renyi Zhang, Yixin Li, Annie L. Zhang, Yuan Wang, Mario J. Molina. Identifying airborne transmission as the dominant route for the spread of COVID-19. P Natl Acad Sci U.S.A. 2020;117(26)14857-14863; DOI: 10.1073/pnas.2009637117

16. World Health Organisation. Coronavirus disease (COVID-19) advice for the public. 2020 [Cited April 2020]. [internet]. Available from: https://www.who.int/emergencies/diseases/novel-coronavirus-2019/advice-forpublic

17. World Health Organisation. Advice on the use of masks in the context of COVID-19. Interim guidance. 2020 [cited June 2020]. [Internet]. Available from URL: https://www.who.int/docs/defaultsource/coronaviruse/temp/who-2019-ncov-ipc-masks-2020-4-eng.pdf? sfvrsn=20ec1cbf_2\&download=true

18. Marijon E, Karam N, Jost D, Perrot D, Frattini B, Derkenne C, Sharifzadehgan A, Waldmann V, Beganton F, Narayanan K, Lafont A. Out-of-hospital cardiac arrest during the COVID-19 pandemic in Paris, France: a population-based, observational study. Lancet Public Health. 202 DOI: 10.1016/s2468-2667(20)30117-1.

19. Harvey P, Higenbottam CV, Owen A, Hulme J, Bion JF. Peer-led training and assessment in basic life support for healthcare students: Synthesis of literature review and fifteen years practical experience. Resuscitation. 2012;83(7):894-899

20. Thorne CJ, Jones CM, Harvey P, Hulme J, Owen A. An analysis of the introduction and efficacy of a novel training programme for ERC basic life support assessors. Resuscitation. 2013 Apr 1;84(4):526-9. 
21. British Heart Foundation. HeartStart Training - CPR. 2014 [cited 16 May 2020]. [internet]. Available from: URL https://www.youtube.com/watch?v=yl-gaQLeAgs

22. Core Team (2020). R: A language and environment for statistical computing. R Foundation for Statistical Computing, Vienna, Austria. URL: https://www.R-project.org/.

23. Resuscitation Council (UK). Lifesaver. [internet]. Available from URL: http://www.life-saver.org.uk/

24. Yeung J, Kovic I, Vidacic $M$ et al. The school Lifesavers study-A randomised controlled trial comparing the impact of Lifesaver only, face-to-face training only, and Lifesaver with face-to-face training on CPR knowledge, skills and attitudes in UK school children. 2017; 120: 138-145

25. British Heart Foundation. CPR training videos. [internet]. Available from URL:

https://www.bhf.org.uk/how-you-can-help/how-to-save-a-life/how-to-do-cpr/cpr-training-videos

\section{Figures}

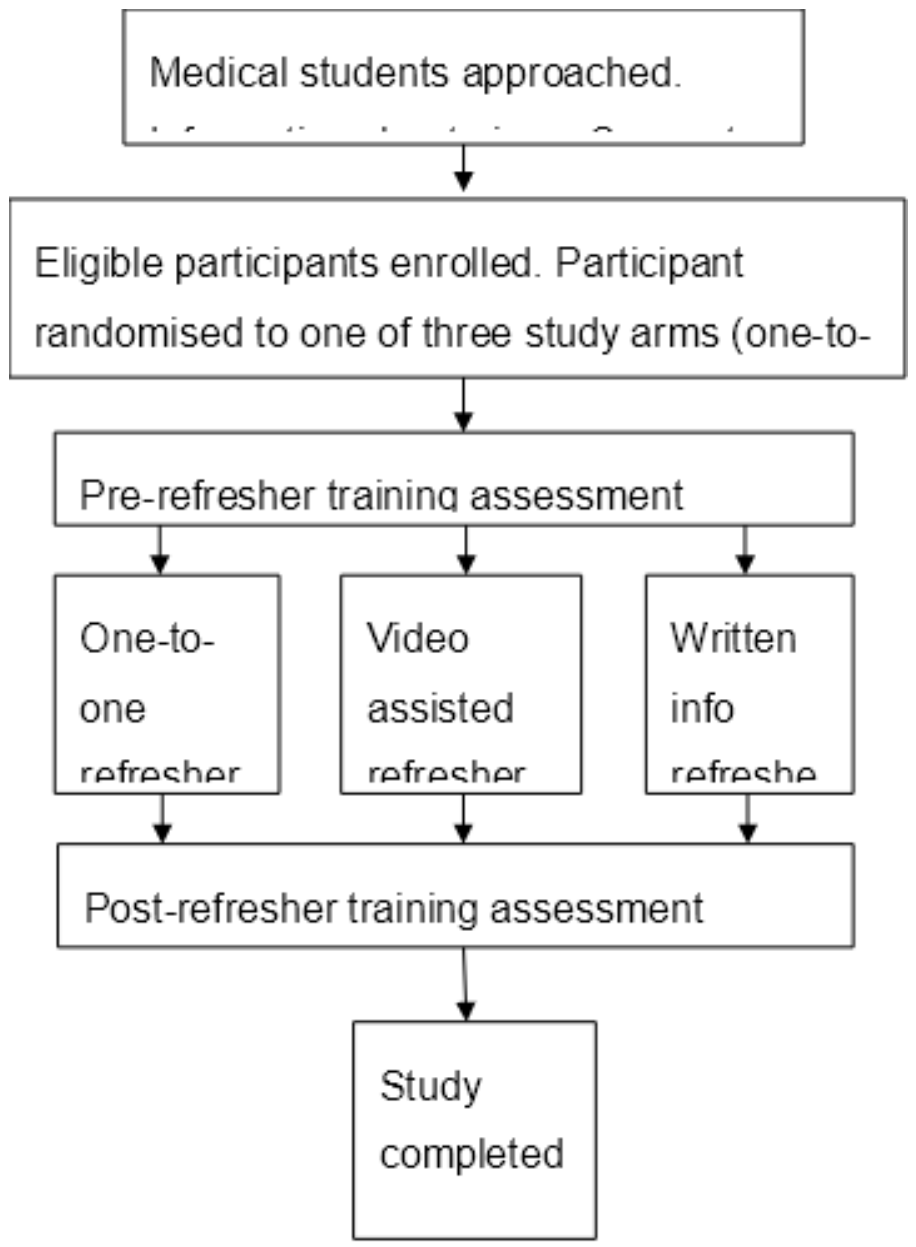

Figure 1

Consort diagram showing recruitment and flow of participants through the study. 

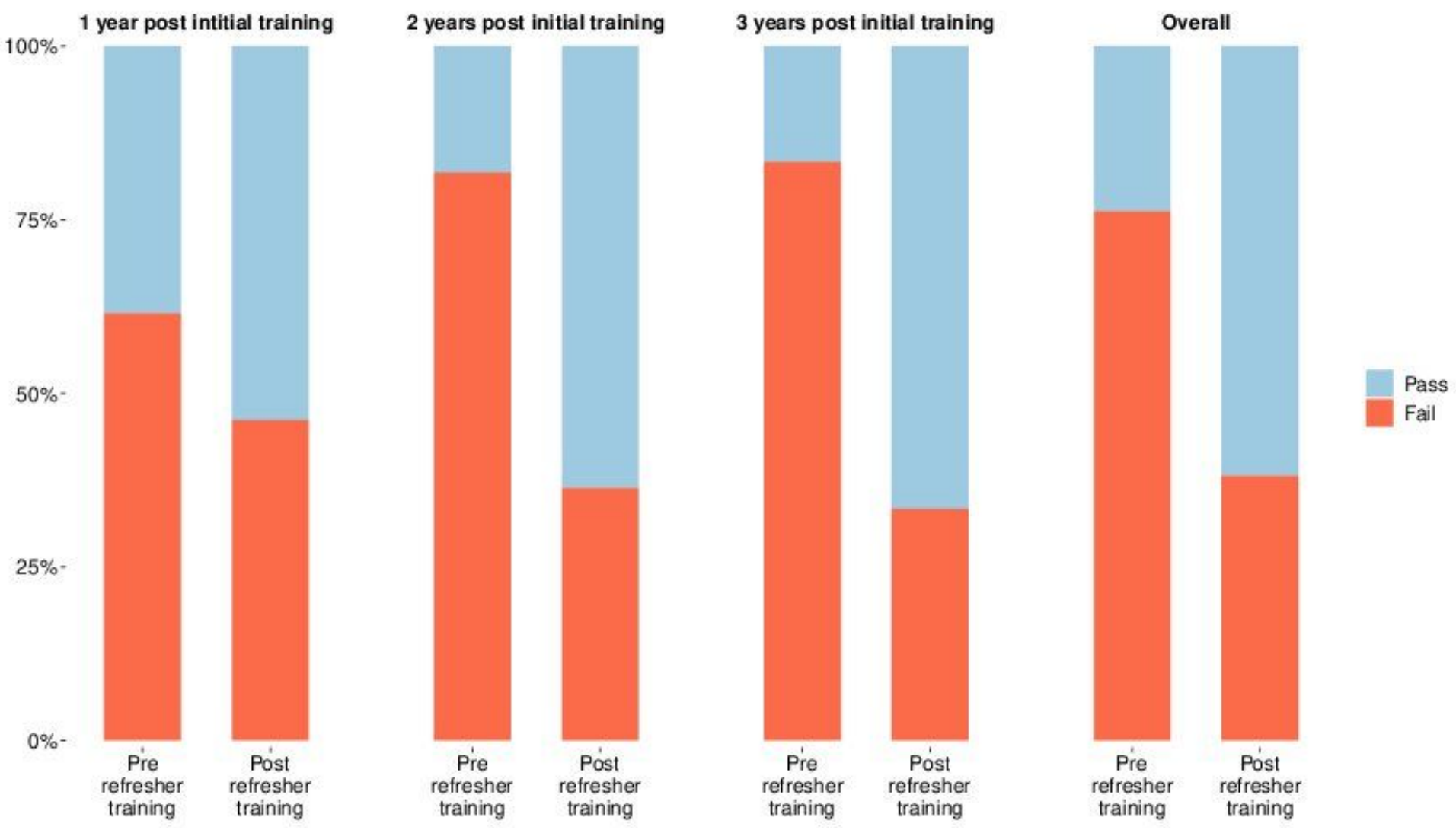

\section{Figure 2}

BLS performance improved following refresher training. 'Pre' indicates pre-refresher training assessment pass rate, 'Post' indicates post-refresher training assessment pass rate. The greatest increase was seen in students who had a larger gap between their initial BLS training, and their refresher training. 